\title{
Allergic Bronchopulmonary Aspergillosis with Repeated Isolation of Nontuberculous Mycobacteria
}

\author{
Takashi Ishiguro ${ }^{1}$, Noboru Takayanagi ${ }^{1}$, Yotaro Takaku ${ }^{1}$, Naho Kagiyama ${ }^{1}$, \\ Yoshihiko Shimizu $^{2}$, Tsutomu Yanagisawa ${ }^{1}$, Yoshinori Kawabata ${ }^{2}$ and Yutaka Sugita ${ }^{1}$
}

\begin{abstract}
A 68-year-old woman without asthma presented with a cough and abnormal shadows on a chest X-ray. Computed tomography showed right middle lobe atelectasis and centrilobular nodules with a tree-in-bud appearance in the other lobes. The patient's sputum repeatedly yielded positive cultures of nontuberculous mycobacteria (NTM); however, no fungi were detected. A transbronchial biopsy showed allergic mucin with eosinophils, although the findings were not diagnostic. We suspected that the patient had pulmonary mycobacteriosis; however, treatment for this condition failed. We then performed thoracoscopy for further evaluation and treatment of the NTM infection. The resected specimen obtained from the right middle lobe exhibited the characteristic findings of allergic bronchopulmonary mycosis without evidence of mycobacterial infection. The administration of corticosteroids and itraconazole resulted in improvement of the patient's condition. The NTM appeared to be simply a coincidental colonization of the resected middle lobe bronchus. The absence of asthma, the inability to isolate fungi and the repeated isolation of NTM made it difficult to differentiate allergic bronchopulmonary aspergillosis from NTM infection.
\end{abstract}

Key words: allergic bronchopulmonary mycosis, aspergillosis, nontuberculous mycobacteria, mycobacteriosis, colonization

(Intern Med 52: 1721-1726, 2013)

(DOI: 10.2169/internalmedicine.52.9537)

\section{Introduction}

Allergic bronchopulmonary aspergillosis (ABPA) is a clinical syndrome that occurs predominantly in asthmatics and is caused by hypersensitivity to the Aspergillus species. ABPA is usually diagnosed based on Rosenberg's criteria (1), which present seven primary diagnostic criteria: episodic bronchial obstruction (asthma), peripheral blood eosinophilia, immediate scratch test reactivity to Aspergillus antigens, precipitating antibodies to Aspergillus antigens, an elevated serum immunoglobulin $\mathrm{E}$ (IgE) concentration, a history of pulmonary infiltrates (transient or fixed) and central bronchiectasis. A diagnosis of ABPA is considered likely if the first six diagnostic criteria are present, and the presence of all seven criteria makes the diagnosis certain. The computed tomography (CT) findings of ABPA consist of central bronchiectasis (typically severe and widespread), mucous plugging, high-density mucus, a tree-in-bud appearance (found in $66.7 \%$ of cases of ABPA (2)), atelectasis, peripheral consolidation or diffuse ground-glass opacity and others $(2,3)$. In contrast, the number of cases of nontuberculous mycobacteria (NTM), which is widely distributed in the environment with high isolation rates worldwide, has been increasing and become a concern in clinical practice. The CT findings of pulmonary NTM infection consist of bronchiectasis, small or large nodules, patchy unilateral or bilateral airspace consolidation, cavitation, scattered airspace nodules, a tree-in-bud appearance, scarring, volume loss and others (4). Bronchiectasis and NTM infection, usually caused by Mycobacterium avium complex, often coexist, and when NTM is repeatedly isolated from the same patient, problems arise in determining whether the isolated NTM indicates an NTM infection or simply colonization. In addi-

${ }^{1}$ Department of Respiratory Medicine, Saitama Cardiovascular and Respiratory Center, Japan and ${ }^{2}$ Department of Pathology, Saitama Cardiovascular and Respiratory Center, Japan

Received for publication December 17, 2012; Accepted for publication March 13, 2013

Correspondence to Dr. Takashi Ishiguro, ishiguro.takashi@ pref.saitama.lg.jp 


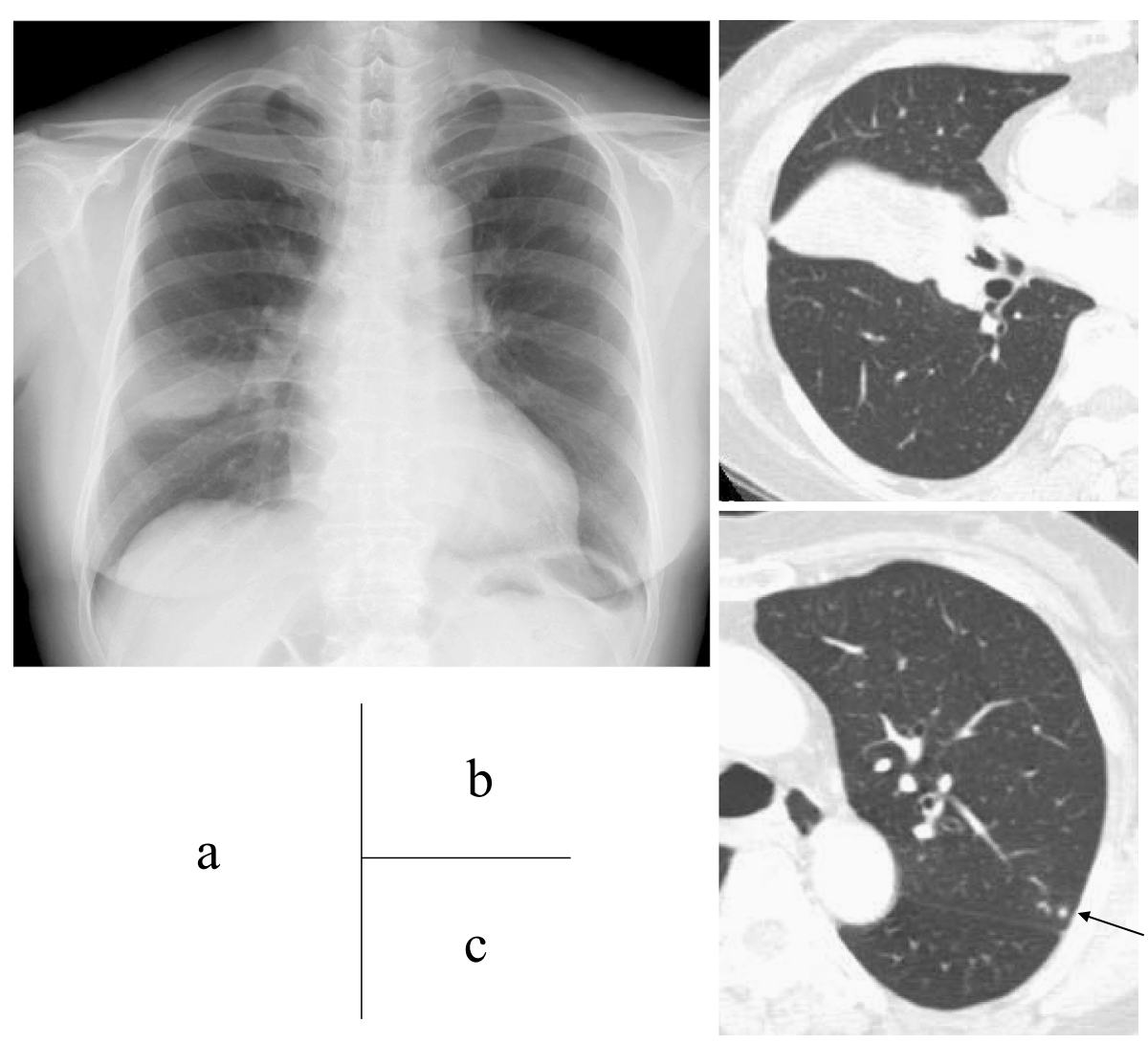

Figure 1. Radiologic findings. Chest $\mathrm{X}$-ray showed a mass in the right middle lung field (a). Chest CT showed subsegmental collapse consequent to bronchial obstruction (b) and centrilobular nodules (arrow) (c).

tion, when isolation of NTM precedes the typical manifestations of ABPA, the patient may be misdiagnosed as having pulmonary mycobacteriosis because pulmonary mycobacteriosis and ABPA have similar radiological features (5). We encountered a patient with ABPA and NTM colonization of an ectatic bronchus due to ABPA. Isolation of NTM preceded the diagnosis of ABPA, and the patient was initially misdiagnosed as having an NTM infection. The diagnosis was corrected based on the histologic findings of the resected lung specimen and the patient's clinical course. We believe that the process that led to a correct diagnosis in this patient will provide clinicians with important clues for differentiating ABPA from NTM infection or colonization.

\section{Case Report}

A 68-year-old woman developed a cough in January 2007 and presented to a local physician. Several antibiotics were administered; however, the patient experienced no improvement. A chest X-ray showed a mass in right middle lung field, and she was referred to our hospital for a further evaluation in March 2007. The patient had never smoked or been exposed to dust, nor had she ever worked in a garden or farm, which would suggest exposure to dense soil or water. She had never experienced periodic paroxysms of dyspnea interspersed with intervals of complete or nearly complete remission, which would suggest asthma. Her height, body weight and temperature were $154 \mathrm{~cm}, 55 \mathrm{~kg}$ and $36.3^{\circ} \mathrm{C}$, respectively. Laboratory tests showed eosinophilia $\left(600 / \mathrm{mm}^{3}\right)$ and an increased IgE level of $480 \mathrm{IU} / \mathrm{mL}$. The erythrocyte sedimentation rate was increased to $34 \mathrm{~mm} /$ $\mathrm{h}$, and the level of C-reactive protein was $0.1 \mathrm{mg} / \mathrm{dL}$. Specific IgE against Aspergillus species was positive; however, antibodies against the Aspergillus species measured using the complement fixation test were negative. Pulmonary function testing (\% predicted) showed a vital capacity of $3.05 \mathrm{~L}$ (106.6\%), a forced vital capacity (FVC) of 2.98 L (104.2\%), a forced expiratory volume in 1 second $\left(\mathrm{FEV}_{1}\right)$ of $2.11 \mathrm{~L}$ $(117.2 \%)$ and an $\mathrm{FEV}_{1} / \mathrm{FVC}$ ratio of $70.8 \%$. In addition, airway responses to a beta-stimulant could not be induced. The patient's sputum yielded positive cultures of Mycobacterium simiae; however, no other microorganisms, including fungi, were cultured. A chest X-ray showed a mass in the right middle lung field (Fig. 1a), and chest CT showed atelectasis of the middle lobes (Fig. 1b) with centrilobular nodules and branching shadows in the left upper lobe (Fig. 1c). No lymphadenopathy or pleural effusion were observed.

We performed bronchoscopy and found the patient's airways to contain thick mucoid secretions. The bronchial washing fluid yielded M. avium, but was negative for fungi. A transbronchial biopsy of the right middle lobe revealed characteristic allergic mucin containing eosinophils, infiltration of eosinophils and neutrophils around the bronchial wall and a necrotizing granuloma, while fungal hyphae were 


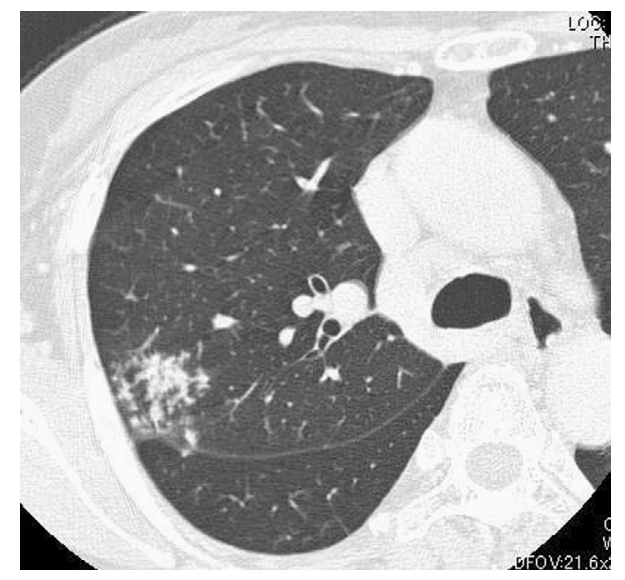

Figure 2. CT findings of the left upper lobe. Chest CT showed centrilobular nodules in the right upper lobe.

absent. Pulmonary NTM infection or ABPA was suspected; however, a definitive diagnosis was not established, and the patient was followed on an outpatient basis. After discharge, her sputum repeatedly yielded positive cultures of $M$. simiae and $M$. intracellulare, and we then suspected her of having a pulmonary NTM infection. We performed another bronchoscopy in which samples of bronchial washing and brushing and the bronchial aspirate specimens all yielded $M$. simiae with the absence of fungi. Clarithromycin, moxifloxacin and sulfamethoxazole-trimethoprim were administered from July 2007 to April 2008; however, the patient's symptoms persisted. In May 2008, the centrilobular nodules in the left upper lobe were found to have decreased, although new centrilobular nodules developed in the right upper lobe in August 2008. M. avium and M. intracellulare were repeatedly cultured from the patient's sputum from August 2008, and rifampicin, ethambutol, clarithromycin and streptomycin were administered; however, the patient's symptoms and chest X-ray findings did not improve, and the centrilobular nodules in the right upper lobe worsened (Fig. 2). Although a diagnosis of ABPA was also suggested by blood eosinophilia, the increased serum IgE level and the bronchoscopic findings, there were concerns that the administration of a systemic corticosteroid targeting ABPA, the diagnosis of which had not been established, could worsen the NTM infection. We then performed thoracoscopy with lobectomy of the right middle lobe and partial resection of the posterior segment of the right upper lobe for further evaluation and treatment of the NTM infection in February 2009.

The right middle lobe lung specimen exhibited ectatic bronchi with high infiltration of eosinophils and lymphocytes in the bronchial wall. Mucous plugs that included eosinophils and Charcot-Leyden crystals were present in the ectatic bronchial lumen (Fig. 3a, b). Fungal hyphae were also found in the mucous plugs (Fig. 3c); however, acid-fast staining revealed no acid-fast bacilli. The histologic appearance of the bronchocentric granulomas was found at the bronchiolar level (Fig. 4a-c). The right upper lobe lung specimen also showed bronchocentric granulomas and fun- gal hyphae along the eosinophilic abscesses at the bronchiolar level. However, no cavitations, caseous necrosis or granulomas with polymorphonuclear leukocytes, which are usually seen in NTM infection, were found in either specimen. Although a clinical diagnosis of bronchial asthma was not established, we diagnosed the patient as having allergic bronchopulmonary mycosis on the basis of the histologic findings (6).

From March 2009, prednisolone at a dose of $20 \mathrm{mg} /$ day and itraconazole at a dose of $200 \mathrm{mg} /$ day were administered, then the corticosteroid was tapered. Subsequently, the patient's cough and sputum production decreased, and the centrilobular nodules observed on CT disappeared. A. niger was cultured from the sputum, and precipitating antibodies against $A$. niger were positive; thus, A. niger was regarded as the causative fungus of the ABPA.

Overall, the frequency of isolation of NTM and A. niger in the present case was as follows. Fifty-nine specimens were obtained for mycobacterial culture. Thirty-seven specimens, including six specimens obtained via bronchoscopy, were collected before the right middle lobe resection, and 11 specimens yielded NTM: $M$. simiae $(\mathrm{n}=5), M$. avium complex $(n=5)$ and M. gordonae $(n=1)$. Following the right middle lobe resection and corticosteroid administration, $22 \mathrm{spu}-$ tum specimens were obtained for mycobacterial culture, and no specimens yielded mycobacteria. In contrast, 47 specimens were obtained for bacterial and fungal cultures. Before the administration of the corticosteroid, 22 specimens were obtained, including six specimens collected via bronchoscopy, and none of them yielded fungi. Following the right middle lobe resection and corticosteroid administration, 25 sputum specimens were obtained for the fungal cultures, three of which yielded A. niger. In September 2011, CT showed disappearance of the centrilobular nodules. The patient is currently being followed on an outpatient basis.

\section{Discussion}

In the present case, NTM was repeatedly isolated from the patient's sputum and bronchoscopy samples. A pulmonary NTM infection was suspected; however, the administration of antimycobacterial therapy was unsuccessful. Based on the histologic findings of the resected lung specimen and the patient's clinical course, she was ultimately diagnosed as having ABPA, and the presence of NTM was regarded as a simple colonization.

Although bronchial asthma is considered to be an essential diagnostic criterion for ABPA $(1,7)$, there were no characteristic symptoms of asthma, and antibodies against the Aspergillus species measured using the complement fixation test were negative. The cases of several patients with ABPA without asthma, in whom the diagnosis was made based on the histological findings and clinical course, have been reported $(8,9)$, several of which have included patients who developed asthma during follow-up. During the follow-up period, diagnostic criteria not present at the time of the in- 


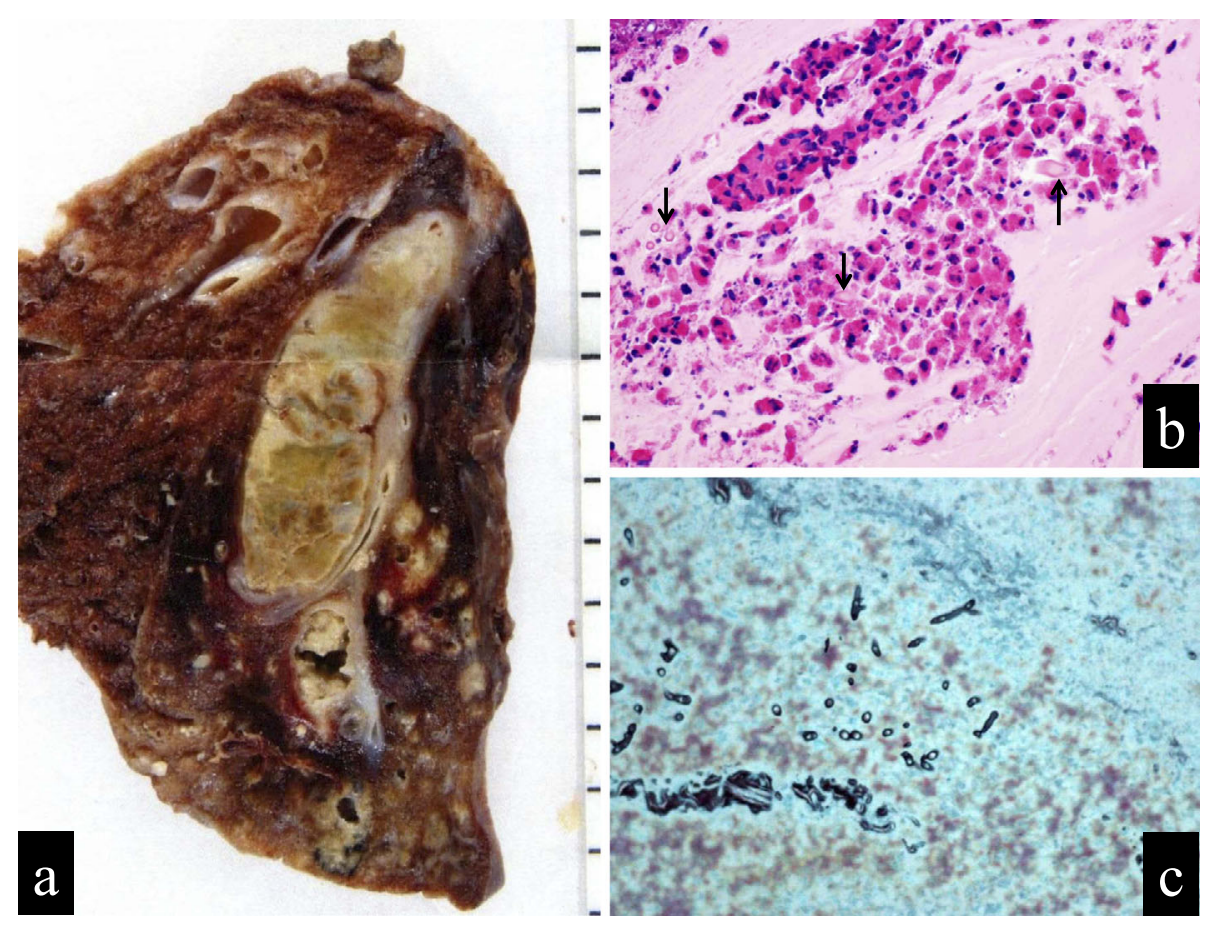

Figure 3. Pathologic findings of the resected specimens obtained from the right middle lobe. a. An examination of the resected specimens showed dilation of the impacted bronchus (arrow). b. Infiltration by eosinophils and Charcot-Leyden crystals was present in the mucous plugs (Hematoxylin and Eosin staining, $\times 60$ ). c. Fragmented septate mycelia were found within the mucous plugs (Grocott stain, $\times 40)$.
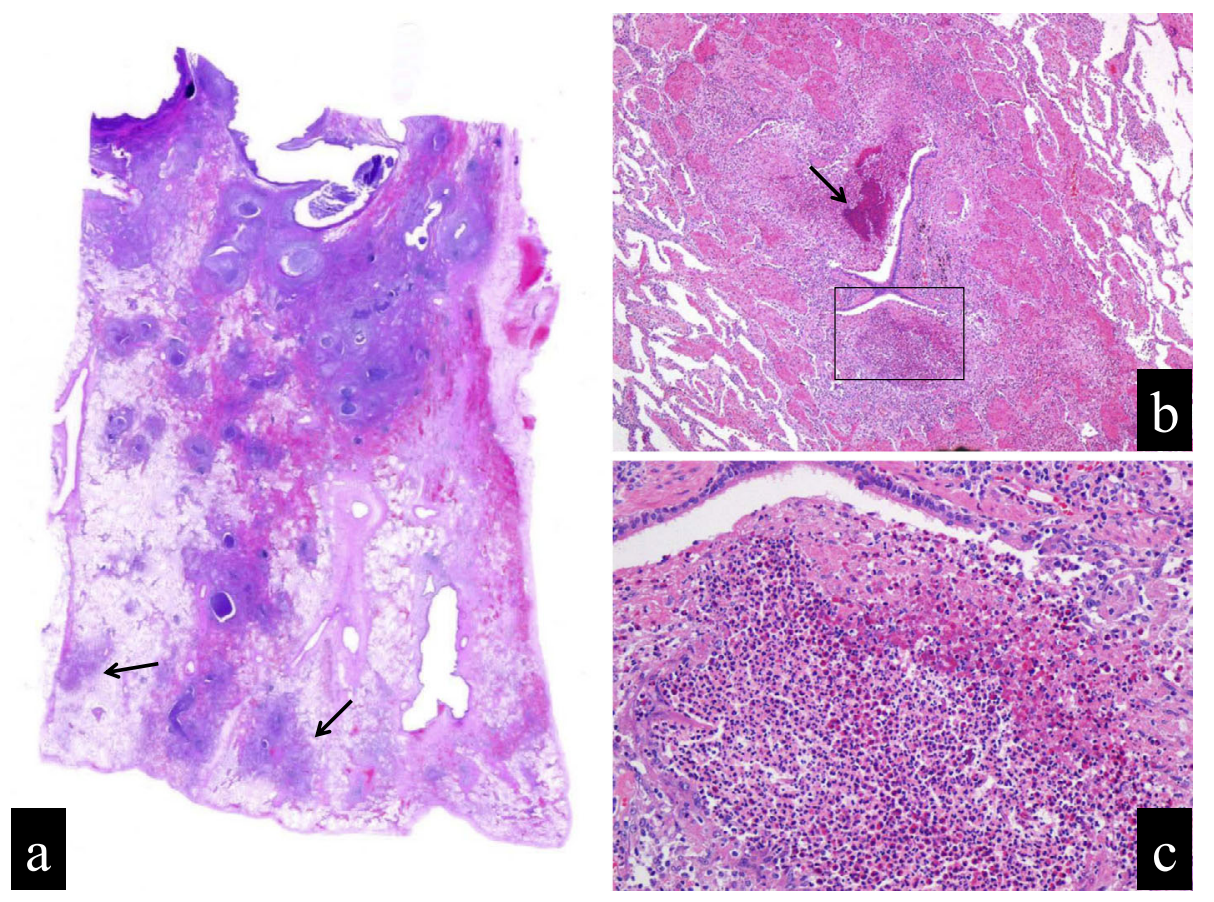

Figure 4. Pathologic findings of mucous plugs and bronchocentric granulomas. a. Mucous plugs were found in the conduction airways, and centrilobular nodules were also detected (arrow) (Hematoxylin and Eosin staining, panoramic view). b. The histological features of the bronchocentric granulomas showed that the respiratory bronchioles were filled with necrotic material (arrow) and inflammatory cells (Hematoxylin and Eosin staining, $\times 4$ ). c. The box in b shows that numerous eosinophils filled the respiratory bronchioles (Hematoxylin and Eosin staining, $\times 20$ ). 
itial diagnosis can develop, which suggests that, especially in the early stage, bronchial asthma may not necessarily be an essential factor for diagnosing ABPA $(10,11)$.

In the present patient, NTM was repeatedly isolated before the resection of the right middle lobe, and the (ATS/ IDSA) criteria for NTM infection, which require the presence of pulmonary symptoms, radiological findings (nodular or cavitary opacities) and isolation of NTM from at least one bronchial washing, were met (12), leading us to suspect an NTM infection. However, there were no evident pathological findings of NTM infection in the resected lung. The centrilobular nodules detected on CT, based on which NTM infection was assumed before surgery, seemed to correspond to bronchocentric granulomas distributed in the bronchiolar lesion pathologically. In addition, sputum cultures for NTM became negative following the right middle lobe resection and administration of a corticosteroid and an antifungal agent. Therefore, it appeared that NTM had simply colonized the resected right middle lobe bronchus.

NTM infection or colonization can occur in persons with impaired pulmonary clearance mechanisms due to chronic lung diseases. To date, several ABPA patients in whom NTM was isolated have been reported (12-16). The isolation of NTM can indicate the presence of both infection and simple colonization. Most previous reports differentiated NTM infection from simple colonization $(13,16)$ according to the ATS/IDSA criteria (12). Mussaffi et al. reported that six of 139 cystic fibrosis patients had ABPA, four of whom developed concomitant NTM infection following the diagnosis of ABPA (13). In some reports $(14,15)$, NTM infection was differentiated from colonization based on histologic findings. Greenberger and Katzenstein reported the case of an 18-year-old woman with ABPA in whom exogenous lipid pneumonia that developed following bronchography had been colonized by $M$. chelonae (14). Although M. chelonae was isolated repeatedly, the resected lung specimen showed no characteristic findings of NTM infection, and the presence of NTM was regarded as colonization. Hanson et al. reported the case of a 14-year-old boy with ABPA who yielded $M$. avium complex in his sputum (15). The resected lung specimen revealed necrotizing granulomatous inflammation with the absence of acid-fast organisms on appropriate stains. The histologic finding of bronchocentric granuloma was also observed; however, this pattern is seen in both ABPA and NTM infection, and the role of the $M$. avium complex was unclear. In any case, NTM can colonize or infect damaged bronchi, such as those seen in patients with advanced ABPA; thus, the significance of NTM isolated from patients with already established ABPA should be considered carefully.

Isolation of NTM preceded the diagnosis of ABPA in the present case. Due to the absence of sufficient proof to diagnose ABPA and the difficulty in differentiating NTM infection from ABPA, our patient unfortunately underwent middle lobe resection via thoracoscopy. The low $\mathrm{FEV}_{1} / \mathrm{FVC}$ ratio observed in our patient indicated a limitation of the air- flow, which was suggestive of airway involvement other than the mucous plugs (17). The patient's eosinophilia, increased serum IgE level, thick mucoid secretion found in her bronchial lumen, histologic findings of characteristic allergic mucin containing eosinophils and infiltration of eosinophils around the bronchial wall suggested ABPA. In addition, performing airway hyperresponsiveness and fraction of exhaled nitric oxide tests (18), positive results of which suggest asthma, may have been informative in understanding the pathophysiology involved in our patient. With more careful consideration of these findings, thoracoscopic resection could have been avoided.

In conclusion, patients with ABPA can have concomitant NTM infection and colonization. It is often difficult to diagnose ABPA, especially when NTM isolation precedes the diagnosis of ABPA, due to the similarities in radiologic features between ABPA and NTM infection. Therefore, the clinical significance of NTM isolated in such patients should be carefully considered.

\section{The authors state that they have no Conflict of Interest (COI).}

\section{References}

1. Rosenberg M, Patterson R, Mintzel R, Cooper BJ, Roberts M, Harris KE. Clinical and immunologic criteria for the diagnosis of allergic bronchopulmonary aspergillosis. Ann Intern Med 86: 405414, 1977.

2. Okada F, Ando Y, Yoshitake S, et al. Clinical/pathologic correlations in 553 patients with primary centrilobular findings on highresolution CT scan of the thorax. Chest 132: 1939-1948, 2007.

3. Webb WR, Müller NL, Naidich DP. Airway diseases. In: HighResolution CT of the Lung. 3rd ed. Lippincott Williams \& Wilkins, Philadelphia, 2001: 467-546.

4. Webb WR, Müller NL, Naidich DP. Diseases characterized primarily by nodular or reticulonodular opacities. In: HighResolution CT of the Lung. 3rd ed. Lippincott Williams \& Wilkins, Philadelphia, 2001: 259-354.

5. Al-Moudi OS. Allergic bronchopulmonary aspergillosis mimicking pulmonary Tuberculosis. Saudi Med J 22: 708-713, 2001.

6. Katzenstein AA. Immunologic lung disease. In: Katzenstein and Askin's Surgical Pathology of Non-neoplastic Lung Disease. 4th ed. Katzenstein AA, Ed. W. B. Saunders, Philadelphia, 2006: 151186.

7. Greenberger PA. Allergic bronchopulmonary aspergillosis. J Allergy Clin Immunol 110: 685-692, 2002.

8. Glancy JJ, Elder JL, McAleer R. Allergic bronchopulmonary fungal disease without clinical asthma. Thorax 36: 345-349, 1981.

9. Hoshino $\mathrm{H}$, Tagaki $\mathrm{S}$, Kon $\mathrm{H}$, et al. Allergic bronchopulmonary aspergillosis due to Aspergillus niger without bronchial asthma. Respiration 66: 369-372, 1999.

10. Greenberger PA, Patterson R. Allergic bronchopulmonary aspergillosis and the evaluation of the patient with asthma. J Allergy Clin Immunol 81: 646-650, 1988.

11. Safirstein BH, D’Souza MF, Simon G, Tai EH, Pepys J. Five-year follow up of allergic bronchopulmonary aspergillosis. Am Rev Respir Dis 108: 450-459, 1973.

12. Griffith DE, Aksamit T, Brown-Elliot BA, et al. An official ATS/ IDSA statement: diagnosis, treatment, and prevention of nontuberculous mycobacterial diseases. Am J Respir Crit Care Med 175: 367-416, 2007.

13. Mussaffi H, Rivlin J, Shalit I, Ephros M, Blau H. Nontuberculous 
mycobacteria in cystic fibrosis associated with allergic bronchopulmonary aspergillosis and steroid therapy. Eur Respir J 25: 324-328, 2005.

14. Greenberger PA, Katzenstein AL. Lipoid pneumonia with atypical mycobacterial colonization. Association with allergic bronchopulmonary aspergillosis. Arch Intern Med 143: 2003-2005, 1983.

15. Hanson G, Flor N, Wells I, Novey H, Galant S. Bronchocentric granulomatosis: a complication of allergic bronchopulmonary aspergillosis. J Allergy Clin Immunol 59: 83-90, 1977.

16. Levy I, Grisaru-Soen G, Lerner-Geva L, et al. Multicenter cross- sectional study of nontuberculous mycobacterial infections among cystic fibrosis patients, Israel. Emerg Infect Dis 14: 378-384, 2008.

17. Kita H, Kobayashi Y, Yamashita K, et al. Bronchial biopsy in allergic bronchopulmonary aspergillosis without clinical asthma. Nihon Kokyuki Gakkai Zasshi 41: 411-415, 2003 (in Japanese, Abstract in English).

18. Alving K, Weitzberg E, Lundberg JM. Increased amount of nitric oxide in exhaled air of asthmatics. Eur Respir J 6: 1368-1370, 1993.

(C) 2013 The Japanese Society of Internal Medicine http://www.naika.or.jp/imonline/index.html 